\title{
Factores de calidad determinantes de la formación práctica de los estudiantes de educación
}

\author{
Sara Serrate González ${ }^{\mathrm{a}}$ \\ Sonia Casillas Martín ${ }^{b}$ \\ Marcos Cabezas González ${ }^{c}$
}

\section{Resumen}

El presente artículo contribuye a conocer y reconocer aquellos factores determinantes de una formación práctica de calidad. Los ejes de interés de la investigación se centran en la satisfacción de los alumnos y la utilidad percibida durante este periodo formativo y la valoración de la evaluación de la materia. Así mismo, se ha estudiado la relación existente con variables como el tipo de centro o las expectativas iniciales. El estudio se sitúa en un enfoque cuantitativo con un diseño de investigación de tipo pre-experimental con pre-test y post-test. El tratamiento de los datos resulta del análisis descriptivo e inferencial, estudiando la relación de las diferentes dimensiones y la influencia o no de algunos factores. La satisfacción general con este período es muy alta, destacando que los estudiantes que se encuentran más satisfechos consideran que las prácticas son más útiles para su formación. Se ha observado también que el centro asignado influye considerablemente en la satisfacción del estudiante. Estos resultados obtenidos permiten tomar decisiones que ayuden a mejorar dicho proceso de prácticas.

Palabras clave: Formación inicial. Prácticas externas. Pedagogía. Satisfacción. Utilidad.

\section{Introducción}

Con la implantación del Espacio Europeo de Educación Superior, uno de los grandes retos es el de poner en práctica un modelo docente centrado más en el aprendizaje y en el desarrollo de competencias, algo que debe superar la

\footnotetext{
a Universidad de Salamanca, Facultad de Ciencias Sociales, Salamanca, España

b Universidad de Salamanca, Facultad de Educación, Salamanca, España

c Universidad de Salamanca, Facultad de Educación, Salamanca, España 
adquisición, por parte de los alumnos, de conocimientos carentes de utilidad más allá de su paso por la universidad. Todos los elementos curriculares deben planificarse y desarrollarse según el tipo de competencias que se desea que adquieran los estudiantes (GARCÍA SANZ; MORILLAS PEDREÑO, 2011), teniendo en cuenta que toda competencia, en su naturaleza pedagógica, debe ser holística, integradora, dinámica y reflexiva; esto implica una formación práctica incorporada al diseño de cada titulación (BARCELÓ CERDÁ; RUIZ CORBELLA, 2015). Así, las universidades europeas han llevado a cabo un proceso de reflexión sobre modelos formativos basados en competencias profesionales y sobre la necesidad de adecuar el prácticum a los criterios organizativos y docentes de la convergencia (ZABALZA BERAZA, 2007; 2011).

Una de las finalidades en la formación de futuros profesionales es la de que los estudiantes aprendan a ejecutar las funciones propias de la profesión para la que se están cualificando. En el logro de este objetivo, el prácticum desempeña un papel fundamental al permitir a los alumnos entrar en contacto directo con el contexto, la realidad laboral y las tareas profesionales, convirtiéndose en un lugar de conexión entre el ámbito laboral y el formativo, en un espacio único de integración y encuentro entre la teoría y la práctica desde el enfoque de las competencias (BARRIOS ARÓS et al., 2014). Así lo expresan Runte Geidel y Lorente García (2014, p. 342) al afirmar que el prácticum: "se convierte en uno de los ámbitos que más interés despierta en este proceso de convergencia, quizás por el papel clave que juega como elemento de tránsito entre la institución universitaria y el campo laboral". Para estas autoras, responde a un periodo estratégico del proceso de socialización profesional, ya que ofrece a los alumnos la ocasión de experimentar en contextos que constituirán su actividad profesional y que por ello representan una oportunidad para favorecer su inclusión en la futura cultura profesional. Idea esta que coincide con la de López y Fernández Herrería (2010) para quienes esta es una materia clave en la formación de cualquier profesional, al ofrecer la oportunidad de experimentar la actividad en contextos y situaciones reales, favoreciendo así la cimentación de su identidad profesional.

La formación práctica del alumnado constituye uno de los ámbitos más relevantes en los nuevos Planes de Estudio de las diferentes titulaciones (GONZÁLEZ SANMAMED; FUENTES ABELEDO, 2011). Con la implantación del EEES, el prácticum nace como un escenario ideal tanto para adquirir y poner en práctica competencias profesionales como para facilitar el tránsito entre el mundo académico y el laboral (MENDOZA LIRA; COVARRUBIAS APABLAZA, 2014). Constituye un marco idóneo que permite al estudiante aplicar los conocimientos teóricos y prácticos adquiridos durante su formación, posibilitando una aproximación global e interdisciplinar a los problemas (RODICIO GARCÍA; IGLESIAS CORTIZAS, 
2011). Una oportunidad única para tomar contacto con el mundo profesional, desarrollar una actividad laboral y contribuir al desarrollo de un conocimiento propio (BLANCO ENCOMIENDA; LATORRE MEDINA, 2008). Y en el campo de la pedagogía, representa una oportunidad para hacer converger el conocimiento de la enseñanza, el conocimiento para la enseñanza y el conocimiento en la enseñanza (GONZÁLEZ SANMAMED; FUENTES ABELEDO, 2011).

Entendemos el Prácticum en Pedagogía como la materia que pone en contacto a los pedagogos con la realidad profesional del mundo de la educación (PAREDES LABRA et al., 2016). En los planes de estudio conducentes al Grado en Pedagogía, esta materia constituye una actividad de naturaleza formativa realizada por los estudiantes y supervisada por las universidades, cuyo objetivo es permitir a los mismos aplicar y complementar los conocimientos adquiridos en su formación académica, favoreciendo la adquisición de competencias que les preparen para el ejercicio de actividades profesionales, faciliten su empleabilidad y fomenten su capacidad de emprendimiento (ESPAÑA, 2011). Se trata de un periodo con identidad propia en la formación universitaria. El alumno cuando cursa esta materia se vincula a un futuro escenario profesional desde donde la universidad garantiza la construcción del conocimiento sobre la práctica, supervisando las tareas realizadas (MARTÍNEZ SERRANO; ALVES MEIRINHOS, 2014).

En este sentido, el prácticum en pedagogía necesita de un contexto organizativo que haga posible la plena activación y la puesta en práctica de las competencias profesionales del grado, de acuerdo con una actuación real, autónoma y que suponga a los alumnos la aceptación de la responsabilidad en su ejecución (VILÁ BAÑOS; ANEAS ÁLVAREZ, 2013). Es necesario que desarrolle en los estudiantes la capacidad de ver por medio de la observación directa; la capacidad de hacer primero en colaboración y después de manera autónoma; la capacidad de ver hacer las actuaciones profesionales para distinguir los distintos enfoques metodológicos; y la capacidad de hacer ver por medio de sus propios desempeños y sus fortalezas y debilidades pre-profesionales (MENDOZA LIRA; COVARRUBIAS APABLAZA, 2014; RUNTE GEIDEL; LORENTE GARCÍA, 2014). En la misma línea, Zabalza Beraza $(2009 ; 2011)$ señala que el prácticum ayuda a los alumnos de pedagogía a ser conscientes de sus fortalezas y debilidades, y poder así analizar su evolución profesional, además de potenciar el desarrollo de competencias metacognitivas.

Todo ello pone de manifiesto la gran importancia de llevar a cabo estudios como el que presentamos, desde el prisma de los estudiantes, que recojan su valoración 
sobre la organización del prácticum, la labor de los tutores universitarios, la tipología centros e instituciones y la dedicación de los tutores profesionales, para establecer el grado de contribución a su desarrollo personal y profesional (MENDOZA LIRA; COVARRUBIAS APABLAZA, 2014). Existen, en esta línea, diversas investigaciones desarrolladas respecto de esta materia en diferentes titulaciones relacionadas con la Educación (BRETONES ROMÁN, 2013; CABRERIZO DIAGO et al., 2010; 2012; GOMES; PALAZZO, 2017; GONZÁLEZ FERNÁNDEZ, 2006; RODICIO GARCÍA; IGLESIAS CORTIZAS, 2011; TURRA DÍAZ; FLORES LUENGO, 2018).

\section{Metodología de la investigación}

Este trabajo se sitúa en un enfoque cuantitativo, con un diseño de investigación de tipo pre-experimental con pre-test y post-test (CAMPBELL; STANLEY, 1963). Se utilizó un método descriptivo-inferencial, por medio del estudio de encuesta electrónica, aplicando el mismo cuestionario antes de comenzar y una vez finalizadas las 240 horas de prácticas en el centro correspondiente. Se ha optado por analizar los datos con esta metodología con el fin de concretar las valoraciones de sus propios protagonistas, cuestión relevante para la mejora y calidad de este proceso de formación profesional.

\subsection{Objetivos}

El estudio realizado pretende conocer en profundidad tanto la satisfacción de los alumnos del Grado en Pedagogía que realizan sus prácticas externas, como su percepción sobre la utilidad de las mismas y los aspectos relacionados con la evaluación de este proceso. El objetivo es conocer las valoraciones otorgadas por los estudiantes, antes de comenzar el periodo de prácticas y comprobar si, una vez finalizadas, existen diferencias. El estudio se realiza en dos promociones de estudiantes consecutivas, buscando, así mismo, comprobar si existen diferencias tras la inclusión de cambios en la materia, de un curso académico al siguiente, y ajustes desde la coordinación en relación, principalmente, a las instituciones colaboradoras y receptoras de alumnos en prácticas, al proceso de adjudicación del estudiante al centro de prácticas y al tipo de materiales de evaluación.

\subsection{Población y muestra}

La población está formada por 120 alumnos que, durante los cursos 14/15 y $15 / 16$, cursaron los 24 créditos de la asignatura de Prácticum. La muestra final (Tabla 1), compuesta por 99 estudiantes, supone un $+4,1 \%$ de error 
Tabla 1. Distribución de la muestra.

\begin{tabular}{|c|c|c|c|c|c|c|c|c|}
\hline & \multicolumn{2}{|c|}{ Sexo } & \multicolumn{3}{|c|}{ Mención } & \multicolumn{3}{|c|}{ Tipo de centro } \\
\hline & Hombre & Mujer & Orientación & $\begin{array}{l}\text { Formación } \\
\text { y gestión }\end{array}$ & $\begin{array}{c}\text { Sin } \\
\text { mención }\end{array}$ & Público & Privado & Concertado \\
\hline f & 14 & 85 & 52 & 14 & 33 & 34 & 17 & 16 \\
\hline \multirow[t]{3}{*}{$\%$} & 14,1 & 85,9 & 52,5 & 14,1 & 33,3 & 50,7 & 25,4 & 23,9 \\
\hline & \multicolumn{3}{|c|}{ Asignación } & \multicolumn{5}{|c|}{ Elección } \\
\hline & $\begin{array}{c}1^{a}-3^{a} \\
\text { Opción }\end{array}$ & Coordinación & $\begin{array}{l}\text { Elegido antes } \\
\text { del proceso }\end{array}$ & Especialidad & Temática & Cercanía & $\begin{array}{l}\text { Responsable } \\
\text { del centro }\end{array}$ & Aprendizaje \\
\hline$f$ & 58 & 5 & 32 & 37 & 20 & 5 & 2 & 29 \\
\hline$\%$ & 58,6 & 5,1 & 32,3 & 37,4 & 20,2 & 5,1 & 2,0 & 29,3 \\
\hline
\end{tabular}

Fuente: Elaboración propia, 2019.

muestral para un nivel de confianza del 95\%, lo que informa suficientemente de su representatividad.

Con relación a la composición de la muestra, es mayor el número de mujeres que el de hombres. Más de la mitad cursaban la mención de Orientación Educativa y el $74,6 \%$ realizaron prácticas en un centro público o concertado. En cuanto al centro de destino, en más de la mitad de los casos se respeta la opción elegida por el estudiante, teniendo en cuenta que el 32,3\% lo tramitaron antes del proceso, y seleccionaron un centro que no se encontraba entre la oferta de la universidad.

En general, los estudiantes seleccionan los centros de prácticas en función de la especialidad que están cursando, pero entre sus prioridades se encuentran también la percepción de la capacidad de aprendizaje que les ofrece el centro y la temática o colectivo con el que se trabaja.

Los centros en los que los estudiantes pueden realizar prácticas se organizan en tres bloques en función de la tipología: ámbito de la orientación escolar, ámbito de la formación y gestión educativa, y ámbito social (organizado en función de los destinatarios con los que trabajan las instituciones).

En la Figura 1 se muestran los tipos de centro en los que los estudiantes realizaron las prácticas externas. Se puede observar que más de un 30\% de los alumnos asisten a departamentos o equipos de orientación educativa, siendo similar la proporción en los dos periodos investigados. 


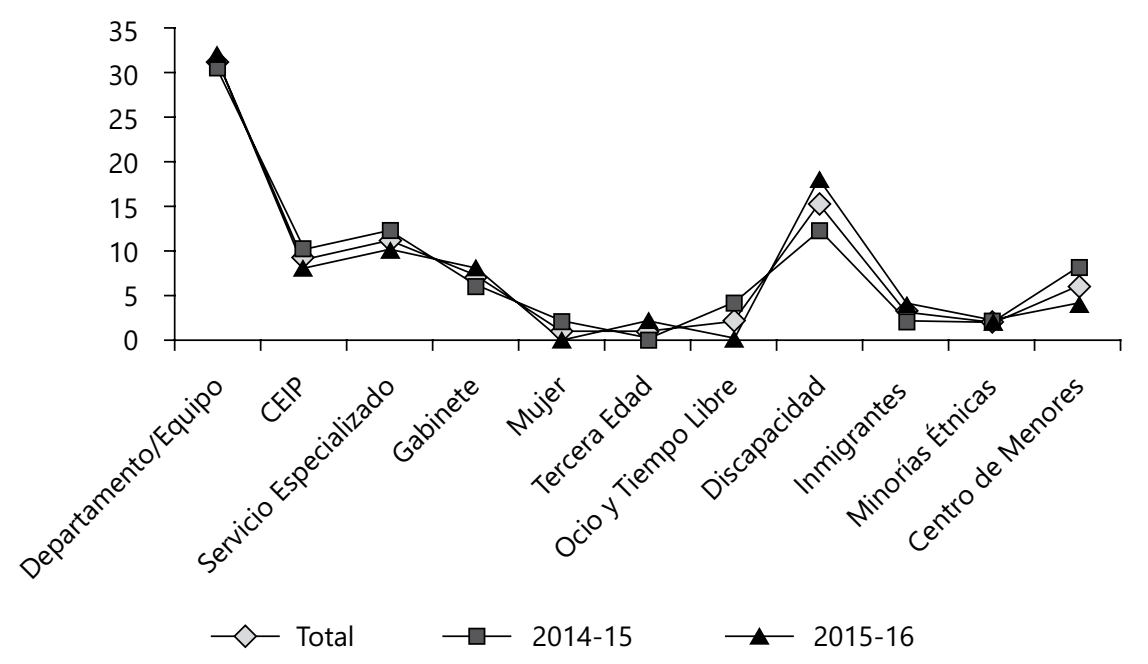

Fuente: Elaboración propia, 2019.

Figura 1. Tipo de centro de prácticas.

\subsection{Instrumento}

El instrumento de recogida de datos es de naturaleza cuantitativa, basado en la metodología de encuesta estructurada, dentro de la que incluimos una escala tipo Likert de 5 puntos con valores que oscilan entre totalmente de acuerdo y totalmente en desacuerdo para la dimensión de satisfacción, y entre nada y mucho para la escala de utilidad. El cuestionario diseñado ad hoc por el equipo de coordinación del Prácticum se aplicó antes y después de que los alumnos realizaran sus prácticas, por medio del servicio Google Drive (RADA, 2012).

La estructura del cuestionario (Tabla 2) consta de 20 ítems incluidos en cuatro bloques de contenidos, siendo el primero los datos sociodemográficos. Cada uno de los ítems fue adaptado a su aplicación pre o post-test. En la Tabla 2 queda recogida la definición operacional de cada una de las dimensiones que componen el instrumento final.

La validez de contenido fue garantizada por medio de la valoración de jueces expertos, académicos especializados tanto en metodología como en el tema de investigación trabajado y con amplia y reconocida experiencia en la coordinación, organización y gestión de la materia Prácticum. Respecto a la consistencia interna del instrumento, la correlación inter-elementos promedio, arroja un coeficiente Alfa de Cronbach de 0.920 en su aplicación pre y de 0,919 en su aplicación post, muy aceptable en ambas aplicaciones. 
Tabla 2. Estructura del cuestionario.

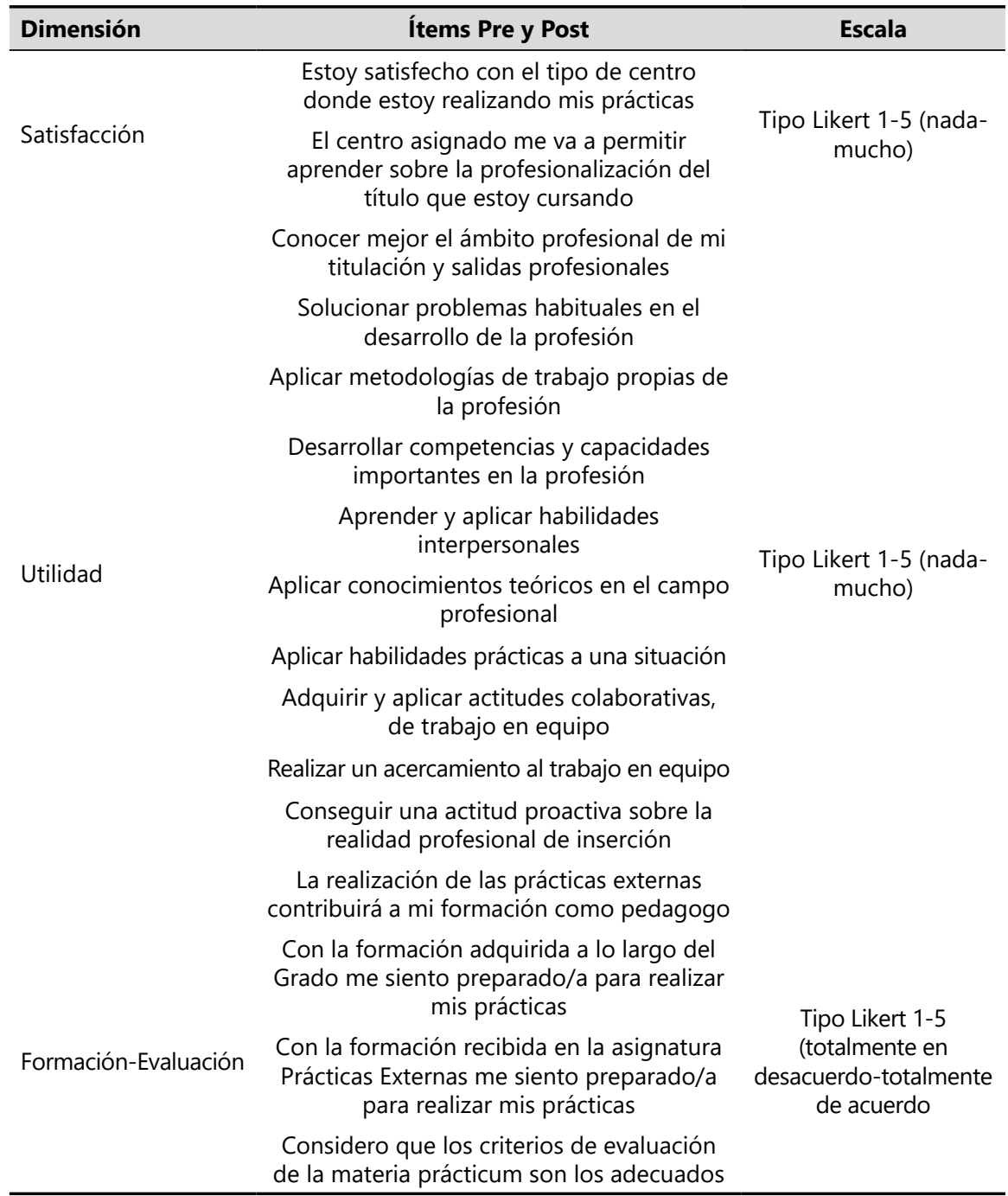

Fuente: Elaboración propia, 2019.

\subsection{Análisis de los datos}

Se utilizó el programa informático SPSS v.21. El tratamiento de los mismos se centró en un análisis descriptivo, a partir del valor de la media sobre las percepciones que tenían los estudiantes antes de comenzar las prácticas, así como las medias sobre la percepción al finalizar dicho periodo. También se 
realizó un análisis de tipo inferencial a partir de una comparación de medias. Una vez comprobados los supuestos paramétricos de normalidad (prueba de Kolmogorov-Smirnov y Shapiro-Wilk) y de Homocedasticidad (prueba de Levene), se optó por la utilización de pruebas no paramétricas, de contraste de hipótesis, en concreto la prueba de rangos de Wilcoxon cuando se trata de muestras relacionadas y la Prueba Kruskal-Wallis para muestras independientes, lo que permitió comprobar la existencia de diferencias estadísticamente significativas en cuanto a las valoraciones recogidas en las dos aplicaciones (pre y post). Del mismo modo, se comprobó la existencia de diferencias en función del curso académico en el que los estudiantes finalizaron el grado. Se presupone que las percepciones de los estudiantes pueden variar en función de diversos factores que afecten a estas valoraciones, por lo que se pretendió comprobar si existían diferencias significativas en función de algunas variables sociodemográficas como la mención que cursan, el tipo de centro, la titularidad del mismo, el proceso de asignación, el motivo de elección, si satisface sus expectativas iniciales, si la plaza se adecua a su perfil, si están satisfechos con el centro y si este les permite aprender la profesionalización del título.

Finalmente, se estudió la relación e influencia de unas dimensiones con otras, bajo la hipótesis de que aquellos estudiantes que consideran que las prácticas son muy útiles son los más satisfechos, así como si los que manifiestan mayor satisfacción son aquellos a los que más les agrada el proceso de evaluación seguido en la asignatura.

\section{Resultados}

\subsection{Dimensión satisfacción}

En general, la satisfacción de los estudiantes con los centros de prácticas es muy positiva (Figura 2). Mantienen la satisfacción inicial con el tipo de centro asignado tras la finalización de las prácticas; sin embargo, esta decae en el post-test con relación a las posibilidades reales de aprendizaje en las instituciones, no encontrándose diferencias estadísticamente significativas al respecto.

Los estudiantes consideran que la plaza asignada se adecua al perfil académico, siendo esta percepción mayor en el segundo año del estudio, cuando se procedió a realizar una nueva distribución y ajuste de centros $(\bar{x}=4.062014-15 ; \bar{x}=4,44$ 2015-16). Cuando se les plantea a los estudiantes, una vez finalizado el periodo de prácticas, si la experiencia se corresponde con sus expectativas iniciales la valoración aumenta de nuevo en el segundo año ( $\bar{x}=3,74$ 2014-15; $\bar{x}=3,84$ 2015-16). Hay que tener en cuenta que en ambos cursos las prácticas satisfacen 


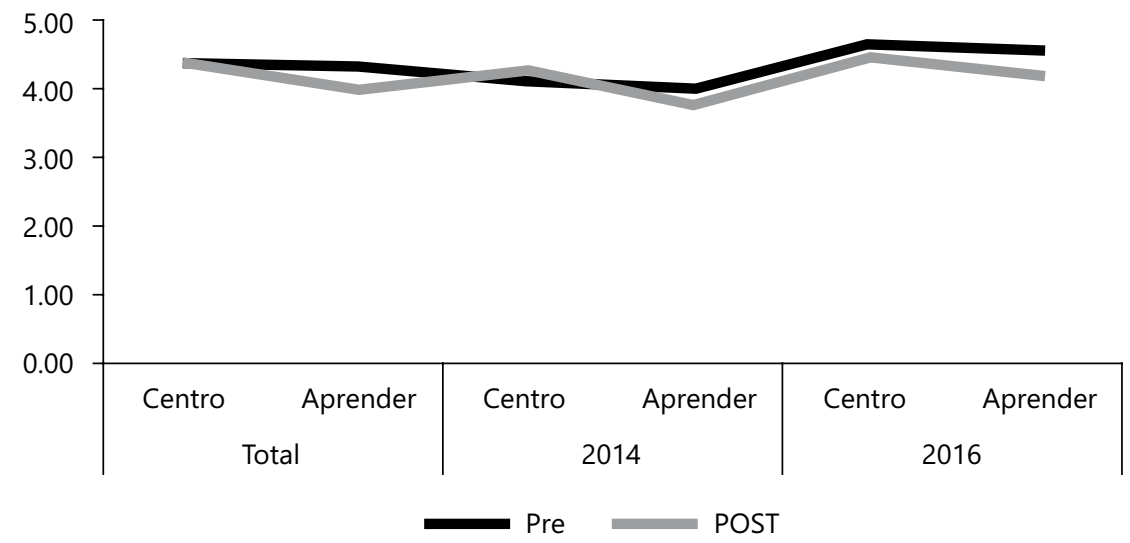

Fuente: Elaboración propia, 2019.

Figura 2. Satisfacción de los estudiantes respecto a los centros de prácticas.

las expectativas iniciales de los estudiantes, aspecto relevante para el mejor aprovechamiento de las mismas.

\subsection{Dimensión utilidad}

El alumnado muestra, de nuevo, una percepción positiva respecto a la utilidad del Prácticum en lo que respecta a su formación. En el análisis conjunto de los resultados obtenidos en los dos años del estudio (Figura 3), las expectativas iniciales son más elevadas que las puntuaciones otorgadas tras finalizar las prácticas, en todos los ítems a excepción de aprender y aplicar habilidades interpersonales propias de mi titulación (ítem 5). Se encontraron diferencias estadísticamente significativas que ponen de manifiesto que la valoración pre-test es más alta que la valoración post-test en los ítems conocer mejor el ámbito profesional de mi titulación y las salidas profesionales (ítem 1) $(\mathrm{r}=-2.68, \mathrm{p} .007)$, aplicar metodologías de trabajo propias de mi futura profesión (ítem 2$)(\mathrm{r}=-2.75, \mathrm{p} .006)$ y la realización de las prácticas externas contribuirá a mi formación como profesional de la pedagogía (ítem 11) $(\mathrm{r}=-2.91, \mathrm{p} .004)$.

Los estudiantes que realizan las prácticas en el segundo curso investigado creen que estas reportan un mayor grado de utilidad que del primer curso académico. Las diferencias estadísticamente significativas halladas en las valoraciones otorgadas en el pre-test y post-test se muestran en la Tabla 3.

También se puede observar que se hallan diferencias estadísticamente significativas al diferenciar las puntuaciones en función del tipo de centro. Teniendo en cuenta 


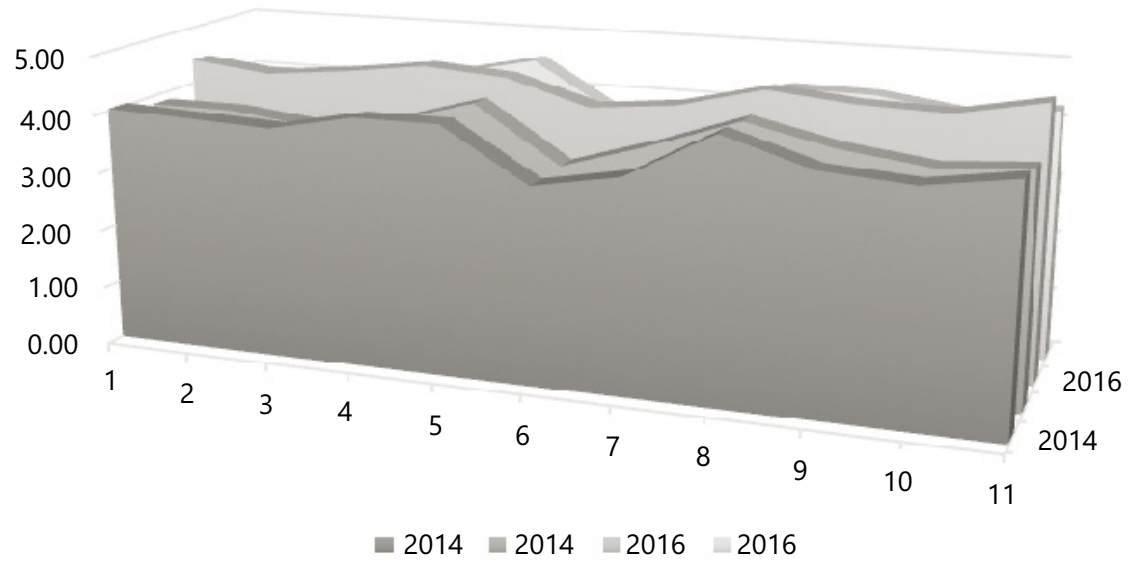

Fuente: Elaboración propia, 2019.

Figura 3. Valoración de la utilidad de las prácticas externas.

las puntuaciones medias, se observa que las mejores valoraciones las conceden aquellos alumnos que realizaron sus prácticas en centros de titularidad concertada, y las peores en centros de titularidad privada.

\subsection{Comparación binomio Satisfacción-Utilidad}

Tras la comparación múltiple realizada entre la dimensión satisfacción y la dimensión utilidad, se confirma la hipótesis de que aquellos estudiantes que se encuentran más satisfechos consideran que las prácticas son más útiles para su formación (Tabla 4). Los estudiantes que piensan que las prácticas realizadas se corresponden con sus expectativas iniciales, consideran antes y después que este periodo les ha permitido conocer mejor el ámbito y salidas profesionales, aplicar metodologías de trabajo propias de la profesión, aplicar conocimientos teóricos y habilidades prácticas a una situación profesional, realizar un acercamiento al trabajo en equipo y contribuir a la formación como profesional de la pedagogía. Tras la realización de las prácticas, quien sigue manteniendo la satisfacción mostrada en sus expectativas iniciales, considera que este periodo le ha permitido solucionar problemas habituales en la profesión, aprender y aplicar habilidades interpersonales, adquirir actitudes colaborativas y conseguir una actitud proactiva.

Los alumnos que manifiestan encontrarse satisfechos con la adecuación del perfil de prácticas consideran antes y después que las prácticas les permiten solucionar problemas habituales de la profesión, aplicar metodologías de trabajo propias de 
Tabla 3. Diferencias en función del curso académico y del tipo de centro.

\begin{tabular}{|c|c|c|c|c|c|c|c|c|c|c|c|c|}
\hline \multirow{3}{*}{$\begin{array}{l}\text { Dimensión } \\
\text { Utilidad }\end{array}$} & \multicolumn{6}{|c|}{ Curso Académico } & \multicolumn{6}{|c|}{ Tipo de centro } \\
\hline & \multicolumn{3}{|c|}{ Diferencia Pre-Test } & \multicolumn{3}{|c|}{ Diferencia Post-Test } & \multicolumn{3}{|c|}{ Diferencia Pre-Test } & \multicolumn{3}{|c|}{ Diferencia Pos-Test } \\
\hline & $x^{2}$ & gl & Sig. & $x^{2}$ & gl & Sig. & $x^{2}$ & gl & Sig. & $x^{2}$ & gl & Sig. \\
\hline Util_1 & - & - & - & - & - & - & 11.131 & 2 & 0.004 & - & - & - \\
\hline Util_2 & - & - & & - & - & - & - & - & - & - & - & - \\
\hline Util_3 & 7.756 & 1 & 0.005 & 3,940 & 1 & .047 & 6.574 & 2 & 0.037 & - & - & - \\
\hline Util_4 & 5.781 & 1 & 0.016 & - & - & - & 6.924 & 2 & 0.031 & - & - & - \\
\hline Util_5 & - & - & - & - & - & - & & & & - & - & - \\
\hline Util_6 & 12.176 & 1 & 0.000 & - & - & - & 6.046 & 2 & 0.049 & 7.173 & 2 & 0.028 \\
\hline Util_7 & 6.966 & 1 & 0.008 & - & - & - & - & - & - & - & - & - \\
\hline Util_8 & - & - & - & - & - & - & - & - & - & - & - & - \\
\hline Util_9 & 5.004 & 1 & 0.025 & 3.886 & 1 & 0.049 & - & - & - & 8.514 & 2 & 0.140 \\
\hline Util_10 & 10.329 & 1 & 0.001 & 4.637 & 1 & 0.031 & 9.540 & 2 & 0.008 & - & - & - \\
\hline Util_11 & 11.055 & 1 & 0.001 & 4.013 & 1 & 0.045 & 11.086 & 2 & 0.004 & 11.239 & 2 & 0.004 \\
\hline
\end{tabular}

Fuente: Elaboración propia, 2019.

la ocupación, realizar un acercamiento al trabajo en equipo y que la realización de las prácticas contribuye a la formación del pedagogo.

Las prácticas resultan útiles en tanto que permiten conocer mejor el ámbito y salidas profesionales, solucionar problemas propios de la profesión, aplicar metodologías de trabajo propias del pedagogo, aprender y aplicar habilidades interpersonales, realizar un acercamiento al trabajo en equipo, conseguir una actitud proactiva y contribuir a la formación del pedagogo para aquellos estudiantes que muestran una mayor satisfacción con el tipo de centro en el que realizan las prácticas de la titulación.

Los estudiantes que desde el inicio se muestran satisfechos al considerar que el tipo de centro les va a permitir aprender afirman, al finalizar el periodo, que les ha resultado una experiencia útil en todos los ítems propuestos, frente a aquellos que se muestran más dubitativos con la idea del aprendizaje asociado al centro de prácticas.

En aquellos casos en los que manifiestan que las prácticas resultan útiles para reflexionar sobre el perfil y trabajo profesional de la titulación cursada, se encuentran diferencias estadísticamente significativas que indican que son los que, además, consideran que la experiencia en el centro de prácticas se corresponde con sus expectativas iniciales (p.000) y que la plaza ocupada se adecúa con el 
Tabla 4. Comparación dimensión Satisfacción-Utilidad.

\begin{tabular}{|c|c|c|c|c|c|c|c|c|c|c|c|c|}
\hline \multirow{4}{*}{$\begin{array}{l}\text { Dimensión } \\
\text { Utilidad }\end{array}$} & \multicolumn{12}{|c|}{ Dimensión Satisfacción } \\
\hline & \multicolumn{6}{|c|}{ Expectativas Iniciales } & \multicolumn{6}{|c|}{ Adecuación al perfil } \\
\hline & \multicolumn{3}{|c|}{ PRE } & \multicolumn{3}{|c|}{ POST } & \multicolumn{3}{|c|}{ PRE } & \multicolumn{3}{|c|}{ POST } \\
\hline & $\mathbf{X}^{2}$ & gl & Sig. & $\mathbf{X}^{2}$ & gl & Sig. & $\mathbf{X}^{2}$ & gl & Sig. & $\mathbf{X}^{2}$ & gl & Sig. \\
\hline Util_1 & 16.211 & 4 & 0.003 & 23.680 & 4 & 0.000 & - & - & - & 24.818 & 4 & 0.000 \\
\hline Util_2 & - & - & - & 26.935 & 4 & 0.000 & 10.855 & 4 & .031 & 16.030 & 4 & 0.003 \\
\hline Util_3 & 10.189 & 4 & 0.037 & 18.568 & 4 & 0.001 & 10.855 & 4 & .028 & 14.509 & 4 & 0.006 \\
\hline Util_4 & - & - & - & 31.617 & 4 & 0.000 & - & - & - & 20.017 & 4 & 0.000 \\
\hline Util_5 & - & - & - & 26.992 & 4 & 0.000 & - & - & - & 14.825 & 4 & 0.005 \\
\hline Util_6 & 20.008 & 4 & 0.000 & 18.848 & 4 & 0.001 & - & - & - & - & - & - \\
\hline Util_7 & 13.880 & 4 & 0.008 & 21.730 & 4 & 0.000 & 10.643 & 4 & 0.031 & - & - & - \\
\hline Util_8 & - & - & - & 9.886 & 4 & 0.042 & - & - & - & - & - & - \\
\hline Util_9 & 13.740 & 4 & 0.008 & 20.581 & 4 & 0.000 & 9.957 & 4 & 0.041 & 10.790 & 4 & 0.029 \\
\hline Util_10 & - & - & - & 24.408 & 4 & 0.000 & 9.475 & 4 & 0.050 & 15.087 & 4 & 0.005 \\
\hline Util_11 & 11.696 & 4 & 0.020 & 32.728 & 4 & 0.000 & 9.648 & 4 & 0.047 & 32.081 & 4 & 0.000 \\
\hline \multirow{4}{*}{$\begin{array}{l}\text { Dimensión } \\
\text { Utilidad }\end{array}$} & \multicolumn{12}{|c|}{ Dimensión Satisfacción } \\
\hline & \multicolumn{6}{|c|}{ Tipo de Centro } & \multicolumn{6}{|c|}{ Centro-Aprendizaje } \\
\hline & \multicolumn{3}{|c|}{ PRE } & \multicolumn{3}{|c|}{ POST } & \multicolumn{3}{|c|}{ PRE } & \multicolumn{3}{|c|}{ POST } \\
\hline & $\mathbf{X}^{2}$ & gl & Sig. & $\mathbf{X}^{2}$ & gl & Sig. & $\mathbf{X}^{2}$ & gl & Sig. & $\mathbf{X}^{2}$ & gl & Sig. \\
\hline Util_1 & 18.223 & 4 & 0.001 & 28.716 & 4 & 0.000 & 22.759 & 4 & 0.000 & 42.069 & 4 & 0.000 \\
\hline Util_2 & 16.975 & 4 & 0.002 & 21.752 & 4 & 0.000 & 21.741 & 4 & 0.000 & 27.083 & 4 & 0.000 \\
\hline Util_3 & 12.946 & 4 & 0.012 & 18.113 & 4 & 0.001 & 24.895 & 4 & 0.000 & 29.705 & 4 & 0.000 \\
\hline Util_4 & 12.140 & 4 & 0.016 & 26.299 & 4 & 0.000 & 16.736 & 4 & 0.002 & 33.932 & 4 & 0.000 \\
\hline Util_5 & 10.981 & 4 & 0.027 & 18.360 & 4 & 0.001 & 10.637 & 4 & 0.031 & 21.877 & 4 & 0.000 \\
\hline Util_6 & 10.943 & 4 & 0.027 & - & - & - & 24.358 & 4 & 0.000 & 20.391 & 4 & 0.000 \\
\hline Util_7 & - & - & - & 15.762 & 4 & 0.003 & 18.892 & 4 & 0.001 & 15.011 & 4 & 0.005 \\
\hline Util_8 & - & - & - & 9.927 & 4 & 0.042 & 15.015 & 4 & 0.005 & 14.306 & 4 & 0.006 \\
\hline Util_9 & 16.480 & 4 & 0.002 & 25.449 & 4 & 0.000 & 15.639 & 4 & 0.004 & 28.840 & 4 & 0.000 \\
\hline Util_10 & 11.724 & 4 & 0.020 & 28.668 & 4 & 0.000 & 17.790 & 4 & 0.001 & 25.709 & 4 & 0.000 \\
\hline Util_11 & 15.513 & 4 & 0.004 & 30.469 & 4 & 0.000 & 34.354 & 4 & 0.000 & 51.565 & 4 & 0.000 \\
\hline
\end{tabular}

Fuente: Elaboración propia, 2019.

perfil profesional (p.000). Del mismo modo, se corresponde con aquellos que se encuentran más satisfechos con el tipo de centro en el que han realizado las prácticas $(0.000)$ y los que consideran que el centro les ha permitido aprender sobre la profesionalización del título (p.000). 


\subsection{Dimensión formación-evaluación}

Un último objetivo planteado en el estudio trata de descubrir si la formación recibida a lo largo del grado y la formación específica de la materia Prácticum, les aporta seguridad de tal forma que se sienten capacitados formativa y competencialmente para realizar las prácticas de la titulación. Los resultados de la aplicación post-test son más elevados que en el post-test en el ítem relativo a la formación de grado recibida, lo que significa que la formación que les ofrece la universidad a lo largo de los tres años de formación anteriores les da la seguridad necesaria para asumir con responsabilidad y competencia profesional sus prácticas, superando incluso sus expectativas iniciales, ya que los datos de la aplicación pre-test muestran resultados más bajos, encontrándose al respecto diferencias estadísticamente significativas $(\mathrm{r}=5.92, \mathrm{p} .000)$ entre la aplicación pre y post-test. Estos resultados permiten considerar que los estudiantes, durante su periodo de prácticas externas en las instituciones, experimentan la puesta en práctica de los conocimientos teóricos adquiridos en la universidad, y esto les permite valorar la importancia que tiene la formación inicial para su futuro desarrollo profesional. No hay que olvidar, no obstante, que no todos los conocimientos adquiridos a lo largo de la formación universitaria van a tener la misma oportunidad de ser experimentados en la práctica y esto tendrá mucho que ver con la singularidad de cada una de las prácticas y centros en los que se realicen.

Por último, hay que señalar que los estudiantes se muestran conformes con los criterios de evaluación (50\% nota propuesta por el centro o institución, $40 \%$ memoria desarrollada y $10 \%$ seminarios teóricos asistidos), aumentando la valoración en el segundo año del estudio.

\section{Discusión y conclusiones}

En este artículo se han expuesto los resultados obtenidos respecto al nivel de satisfacción de los alumnos del Grado en Pedagogía sobre sus prácticas externas, así como su percepción sobre la utilidad de las mismas y los criterios de evaluación del proceso.

A partir de los resultados obtenidos en la dimensión satisfacción y teniendo en cuenta otras investigaciones sobre el tema (HERNÁNDEZ-PIZARRO et al., 1999; CAMPILLO ALHAMA; QUILES SOLER, 2014; LASA ARISTU, 2006; PÉREZ LORENZO et al., 2013) podemos concluir que, en general, la experiencia vivida se correspondió con sus expectativas iniciales, a diferencia de otros estudios como el realizado por González Fernández (2006) en el que se comprobó la existencia de una desproporción entre las expectativas iniciales (altas) y la satisfacción final (media-baja). 
Los estudiantes consideran que la plaza que se les asignó se adecua al perfil académico de un pedagogo, siendo esta percepción mayor en el segundo año del estudio que en el primero, a partir del ajuste en el que se buscó que los centros dispusieran de pedagogos como tutores profesionales y que el estudiante, durante el tiempo de estancia en formación práctica, cumpliera funciones propias de la titulación. De la misma manera, mantienen su satisfacción inicial con el tipo de centro que se les asignó, una vez que finalizaron su periodo formativo. Sin embargo, esta satisfacción decae en el post-test con relación a las posibilidades reales de aprendizaje en los centros, resultado este corroborado por otras investigaciones como la de Lasa Aristu (2006). Si bien es cierto, se trabaja antes y después de iniciar el periodo de prácticas con los estudiantes para que comprendan que el centro de prácticas es una experiencia formativa única, limitada y que no corresponde con la totalidad de ámbitos en los que puede ejercer el profesional de la pedagogía. En este sentido, los resultados de nuestra investigación van en esta línea: los estudiantes afirman al finalizar las prácticas que iniciaron este periodo con unas altas expectativas que posteriormente decaen, no porque en el centro asignado no se realice un aprendizaje de calidad, sino porque en una única institución no pueden llegar a conocer el trabajo del pedagogo en todo el elenco de espacios profesionales que tiene asignados.

En definitiva, las características del centro, sus posibilidades de aprendizaje, si les permite aprender la profesionalización del centro, si les satisface y cumple con sus expectativas, etc., son aspectos cruciales que condicionan la mayor o menor satisfacción del alumno hacia las prácticas. Todo ello nos lleva a afirmar que esta satisfacción supone un aspecto relevante para el mejor aprovechamiento de este periodo formativo.

Si tenemos en cuenta la dimensión utilidad, de nuevo, los estudiantes muestran una valoración positiva de este periodo formativo práctico, al igual que en otras investigaciones como la de Hernández-Pizarro et al. (1999), siendo esta mayor en la muestra del segundo año investigado.

En general, aquellos que se consideran más satisfechos con el tipo de centro son los que manifiestan una mayor utilidad de las mismas. Solamente en lo relativo al aprendizaje y aplicación de habilidades interpersonales propias de la titulación, las expectativas iniciales fueron inferiores. Es necesario plantearse que los alumnos esperan, tras la estancia en prácticas, dominar a la perfección su profesión; sin embargo, cuando finalizan las mismas, se dan cuenta de que la realidad es demasiado compleja como para dominarla tan fácilmente, que no existen recetas, y que la casuística y las situaciones son tan diversas que 
no es sencillo controlar la profesión en cuatro meses. Esto, en ocasiones, les desanima y puede explicar que se vea mermada su valoración hacia la utilidad de dichas prácticas.

Por otra parte, se encuentran diferencias estadísticamente significativas en función del tipo de centro. Las mejores valoraciones se corresponden con centros de titularidad concertada y las peores con centros de titularidad privada. Este resultado contrasta con los obtenidos en investigaciones en las que los alumnos que mejores valoraciones otorgan son los que han realizado sus prácticas en un centro de carácter privado (GONZÁLEZ RIAÑO; HEVIA ARTIME, 2011).

En definitiva, las prácticas resultan útiles en tanto que permiten conocer mejor el ámbito y salidas profesionales, solucionar problemas de la profesión, aplicar metodologías de trabajo propias del pedagogo, aprender y aplicar habilidades interpersonales, realizar un acercamiento al trabajo en equipo, conseguir una actitud proactiva y contribuir a la formación del pedagogo. Idea esta refrendada en otros estudios como el de Pérez Lorenzo et al. (2013).

Si relacionamos la satisfacción con la utilidad, podemos concluir que aquellos estudiantes que consideran que las prácticas realizadas se correspondieron con sus expectativas iniciales, manifiestan antes y después que este periodo les ha permitido adquirir diversas competencias. Como señalan González Riaño y Hevia Artime (2011), los alumnos están bastante de acuerdo con la utilidad del prácticum como instrumento formativo; sin embargo, otros estudios muestran la rotunda negativa de los alumnos ante el hecho de que las prácticas no han contribuido a clarificar su perfil profesional (HERNÁNDEZ-PIZARRO et al., 1999).

Centrándonos en la dimensión evaluación-formación, tras realizar las prácticas, se obtienen puntuaciones menores que antes de las mismas. Esto puede explicarse por la duración de las mismas (desde que reciben la formación para el prácticum hasta que finalizan la estancia trascurren 5 meses), al compaginar la formación práctica en centros con la formación teórica en la universidad complica la capacidad de los estudiantes para realizar tareas en otro lugar diferente al de la ubicación física de la universidad. Sin embargo, los alumnos, tras finalizar su periodo de prácticas, valoran mejor la formación recibida a lo largo de los cuatro años del grado, superando sus expectativas iniciales, a diferencia de otras investigaciones (MENDOZA LIRA; COVARRUBIAS APABLAZA, 2014) en las que los estudiantes opinan que los conocimientos aprendidos en la universidad no son útiles para adquirir y desarrollar competencias profesionales durante sus 
prácticas. Esto es indicativo de que el estudiante, una vez que se enfrenta a la realidad profesional y, por ello, a actuar como sujeto pre-profesional, al verse implicado en la acción y en un contexto profesional, valora mejor la formación que ha recibido a lo largo de los cuatro años de grado, pues realmente es cuando hace un análisis de todo el conocimiento adquirido y esto le permite desenvolverse en el campo profesional.

Al igual que en otras investigaciones (GONZÁLEZ RIAÑO; HEVIA ARTIME, 2011), los alumnos se muestran conformes con los criterios de evaluación seguidos en la materia aumentando la valoración respecto a la memoria en el segundo año de la investigación, pues se realizaron modificaciones respecto al primer año, entre las que se simplificó el modelo de memoria exigida, centrando el interés en el diario de actividades realizadas durante su estancia y en la reflexión sobre las mismas, lo que invita a la investigación y a la reflexión sobre la propia práctica, metodología denominada investigación-acción. Estos cambios fueron aceptados positivamente por los estudiantes.

Para terminar, destacar la importancia de evaluar y tomar decisiones respecto a los procesos de prácticas es importante y útil, puesto que los estudiantes muestran mejores resultados, mejores valoraciones y percepciones, a la hora de enfrentarse a las prácticas y también una vez que las finalizan, de un año para otro. A partir del estudio realizado, podemos extraer una serie de reflexiones para tener en cuenta de cara a organizar la formación práctica de los estudiantes de grado con garantías de calidad y, aumentar así la satisfacción de los estudiantes respecto al proceso de formativo, ofreciendo mayores posibilidades de aprovechamiento de este periodo que repercuta de forma positiva en el futuro laboral de los estudiantes (Figura 4).

Se debe realizar, en primer lugar, un ejercicio exhaustivo de selección de centros de formación práctica de calidad, teniendo en cuenta, como factores de calidad, disponer de tutores pedagogos o que desempeñen las funciones propias de este profesional, ofrecer una adecuada distribución temporal de las funciones del alumno en prácticas $\mathrm{y}$, en la medida de las posibilidades, tener en cuenta las preferencias de los estudiantes antes de asignar centro en prácticas.

En segundo lugar, es importante mantener la satisfacción durante el proceso formativo, cuyos factores de calidad radican en fomentar un aprendizaje centrado en el alumno, propiciar un espacio de interacción profesional ofreciendo al mismo tiempo apoyo académico y utilizar procesos combinados de evaluación que permitan conseguir mejores resultados. 


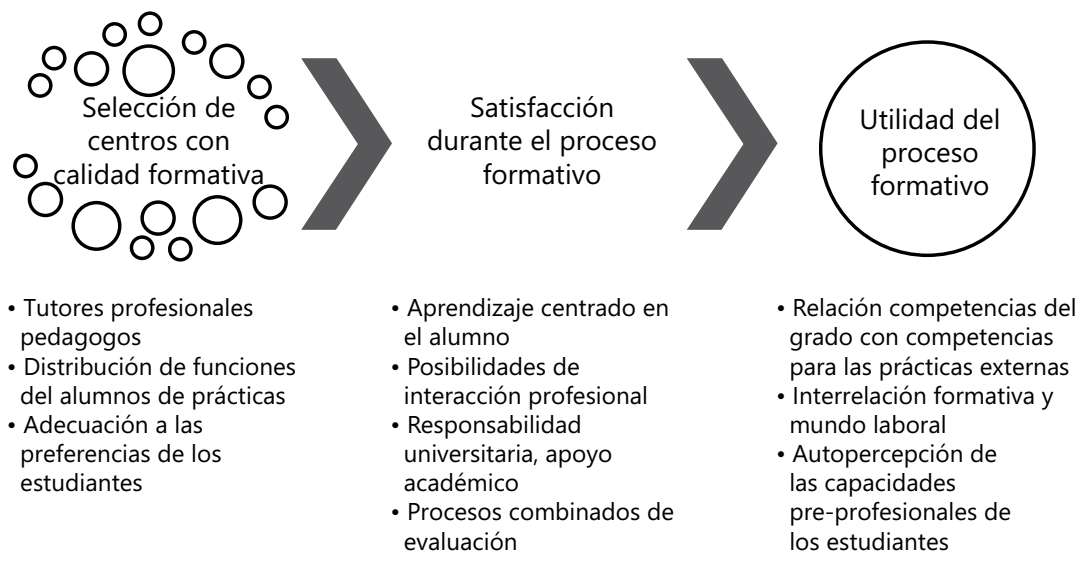

Fuente: Elaboración propia, 2019.

Figura 4. Factores de calidad determinantes de la formación práctica de los estudiantes de educación.

En último lugar, es necesario garantizar la utilidad y el futuro aprovechamiento del proceso formativo. En este caso, los factores de calidad se hallan asociados a la capacidad de relacionar las competencias del Grado en Pedagogía con las competencias exigidas para esta materia, del mismo modo que lograr una interrelación entre la academia, es decir, la formación teórica impartida en la universidad y el mundo laboral, en cuanto a las funciones reales que desempeña un pedagogo en su puesto laboral. Estos dos aspectos favorecen la percepción de los estudiantes en cuanto a sus propias capacidades, al tener que poner en práctica lo aprendido y lo que el mundo profesional les exige. 


\section{Fatores de qualidade determinando a formação prática de estudantes de educação}

\section{Resumo}

Este artigo contribui para conhecer os fatores determinantes de um treinamento prático de qualidade. Os eixos de interesse da pesquisa se concentram na satisfação dos alunos e na utilidade percebida durante o período de treinamento e na avaliação do assunto. Da mesma forma, o relacionamento existente com variáveis, como o tipo de centro ou as expectativas iniciais, foi estudado. O estudo baseia-se em uma abordagem quantitativa com um projeto de pesquisa pré-experimental com pré-teste e pós-teste. $O$ tratamento dos dados resulta da análise descritiva e inferencial, estudando a relação das diferentes dimensões e a influência ou não de alguns fatores. A satisfação geral com esse período é muito alta, observando que os alunos mais satisfeitos consideram que as práticas são mais úteis para o treinamento. Observou-se, também, que o centro atribuído tem uma influência considerável na satisfação dos estudantes. Esses resultados nos permitem tomar decisões que ajudam melhorar esse processo de prática.

Palavras-chave: Treinamento inicial. Práticas externas. Pedagogia. Satisfação. Utilidade

\section{Determinant quality factors in the practical training of education students}

\section{Abstract}

The aim of this article is to help us to learn and recognize the factors that determine quality practical training. The research focused on student satisfaction and perceived usefulness during the training period and students'opinion of the assessment of the subject. The relation between variables such as the type of school or the students'initial expectations regarding the process was also studied. The study employs a qualitative approach, with a pre-experimental research design using pre-test and post-test. Data analysis was descriptive and inferential, examining the relations between the different dimensions and the influence or not of certain factors. It was confirmed that students' general satisfaction with this training period was very high, and it can be highlighted that the most satisfied students were also the ones who found the practical training to be most useful for their education. It was also observed that the school they were assigned to considerably influenced student satisfaction. The findings obtained will allow us to make decisions that help to improve practical training.

Keywords: Initial training. Student teaching. Education degree. Satisfaction. Usefulness. 


\section{REFERENCIAS}

BARCELÓ CERDÁ, M. L.; RUIZ CORBELLA, M. Las competencias profesionales del maestro de primaria desde la perspectiva del tutor del centro de prácticas. Revista Fuentes, n. 17, p. 17-39, dic. 2015. https://doi.org/10.12795/revistafuentes.2015.i17.01

BARRIOS ARÓS, C.; IRANZO GARCÍA, P.; TIERNO GARCÍA, $J$. $M$. Prácticum y trabajo fin de máster como ejes profesionalizadores: análisis de un máster erasmus mundus. Universitad Terraconensis: Revista de Ciències de l'Educació, n. 2, p. 49-73, 2014. https://doi.org/10.17345/ute.2014.2.460

BLANCO-ENCOMIENDA, F. J.; LATORRE-MEDINA, M. J. La apuesta por la excelencia en la formación práctica universitaria de futuros profesores. Estudios sobre Educación, n. 15, p. 7-29, 2008.

BRETONES ROMÁN, A. El prácticum de magisterio en educación primaria: una mirada retrospectiva. Revista Complutense de Educación, v. 24, n. 2, p. 443-71, 2013. https://doi.org/10.5209/rev_RCED.2013.v24.n2.42088

CABRERIZO DIAGO, J.; CASTILLO ARREDONDO, S.; RUBIO ROLDÁN, M. J. El prácticum en los grados de pedagogía, de magisterio y de educación social. Madrid: Pearson Educación, 2010.

CABRERIZO DIAGO, J. et al. El prácticum del máster en formación del profesorado en ESO, bachillerato, FP y EOI. Madrid: Pearson Educación, 2012.

CAMPBELL, D. T.; STANLEY, J. C. Experimental and quasi-experimental designs for research. Chicago, IL: Rand-McNally, 1963.

CAMPILLO ALHAMA, C.; QUILES SOLER, M. C. Evaluación del prácticum en la formación de posgrado en los estudios de comunicación desde una orientación profesional y discente. Historia y Comunicación Social, v. 19, n. esp., p. 327-40, ene. 2014. https://doi.org/10.5209/rev_HICS.2014.v19.44961

ESPAÑA. Real decreto No 1.707/2011, de 18 de noviembre de 2011. Se regulan las prácticas académicas externas de los estudiantes universitarios. Boletin Oficial del Estado, 10 dic. 2011. 
GARCÍA SANZ, M. P.; MORILLAS PEDREÑO, L. R. La planificación de evaluación de competencias en educación superior. Revista Electrónica Interuniversitaria de Formación del Profesorado, v. 14, n. 1, p. 113-24, 2011.

GOMES, C. A.; PALAZZO, J. Factores de atracción y de rechazo a la carrera del magisterio: un análisis de estudiantes y egresados de programas de licenciatura. Ensaio: Avaliação e Políticas Públicas em Educação, Rio de Janeiro, v. 25, n. 94, p. 90-113, ene./mar. 2017. https://doi.org/10.1590/s0104-40362017000100004

GONZÁLEZ FERNÁNDEZ, N. Evaluación y mejora del practicum en las titulaciones de ciencias de la educación de la UPV/EHU. Revista de Psicodidáctica, v. 11, n. 1, p. 145-58, 2006.

GONZÁLEZ RIAÑO X. A.; HEVIA ARTIME, I. El prácticum de la licenciatura de pedagogía: estudio empírico desde la perspectiva del alumnado. Revista de Educación, n. 354, p. 209-36, ene./abr. 2011.

GONZÁLEZ SANMAMED, M.; FUENTES ABELEDO, E. J. El prácticum en el aprendizaje de la profesión docente. Revista de Educación, n. 354, p. 47-70, ene./abr. 2011.

HERNÁNDEZ-PIZARRO, M. A. C. et al. El prácticum de pedagogía: evaluación crítica de la opinión del alumnado. Revista Electrónica Interuniversitaria de Formación del Profesorado, v. 2, n. 1, p. 639-52, 1999.

LASA ARISTU, A. El prácticum en la facultad de psicología de la UNED: resultados de la evaluación de su experiencia piloto en el itinerario de la psicología clínica. Acción Psicológica, v. 4, n. 1, p. 25-36, 2006. https://doi.org/10.5944/ap.4.1.490

LÓPEZ, M. C.; FERNÁNDEZ HERRERÍA, A. Reflexiones y demandas de egresados de la Universidad de Granada sobre la formación práctica: aportes para la mejora del espacio europeo de educación superior. Archivos Analíticos de Políticas Educativas, v. 18, n. 31, p. 1-28, 2010.

MARTÍNEZ SERRANO, M. C.; ALVES MEIRINHOS, M. F. El prácticum en la movilidad erasmus: colaboración entre instituciones de enseñanza superior. Revista Electrónica de Investigación y Docencia, n. 11, p. 91-106, ene. 2014. 
MENDOZA-LIRA, M.; COVARRUBIAS-APABLAZA, C. G. Valoración del prácticum de los grados del magisterio desde la perspectiva de sus estudiantes. Revista Electrónica Educare, v. 18, n. 3, p. 111-42, 2014. https://doi.org/10.15359/ree.18-3.7

PAREDES-LABRA, J.; ESTEBAN-MORENO R. M.; FERNÁNDEZ-PRIETO, M. S. El prácticum de maestro en las voces de sus tutores: balance del plan 2010 en la UAM. Revista Complutense de Educación, v. 27, n. 1, p. 161-78, 2016. https://doi.org/10.5209/rev_RCED.2016.v27.n1.45673

PÉREZ-LORENZO, B; VIANELLO OSTI, M.; REYES PACIOS, A. El practicum del grado en información y documentación: la experiencia en la UC3M. Anales de Documentación, v. 16, n. 2, p. 1-17, 2013. https://doi.org/10.6018/analesdoc.16.2.180381

RADA, V. D. Ventajas e inconvenientes de la encuesta por internet. Papers, v. 97 , n. 1, p. 193-223, 2012. https://doi.org/10.5565/rev/papers/v97n1.71

RODICIO GARCÍA, M. L.; IGLESIAS CORTIZAS, M. La formación en competencias a través del prácticum: un estudio piloto. Revista de Educación, n. 354, p. 99-124, ene./abr. 2011.

RUNTE GEIDEL, A.; LORENTE GARCÍA, R. Evaluación del prácticum de pedagogía: cambios hacia el espacio europeo de educación superior. Estudios Pedagógicos, Valdivia, v. 40, n. 2, p. 341-60, 2014. https://doi.org/10.4067/S0718-07052014000300020

TURRA-DIAZ, Omar; FLORES-LUEG, Carolina. La formación práctica desde las voces del estudiantado de pedagogía. Ensaio: aval. pol. públ. Educ., Rio de Janeiro , v. 27, n. 103, p. 385-405, jun. 2019. http://dx.doi.org/10.1590/s0104-40362018002601517.

VILÁ BAÑOS, R.; ANEAS ÁLVAREZ, A. Los seminarios de práctica reflexiva en el prácticum de pedagogía de la Universidad de Barcelona. Bordón, v. 65, n. 3, p. 165-81, 2013.

ZABALZA BERAZA, M. A. El prácticum y las prácticas en empresas en las coordenadas de la convergencia. In: IGLESIAS FORNERO, M. L. et al. (Coords.). El prácticum en el nuevo contexto del espacio europeo de educación superior. Santiago de Compostela: Universidad de Santiago de Compostela, 2007. p. 63-87. 
ZABALZA BERAZA, M. A. Prácticum y formación: ¿en qué puede formar el practicum? In: RAPOSO VIGAS, M. et al. (Coords.). El prácticum más allá del empleo: formación vs. training. Santiago de Compostela: Universidad de Santiago de Compostela, 2009. p. 45-65.

. El prácticum en la formación universitaria: estado de la cuestión.

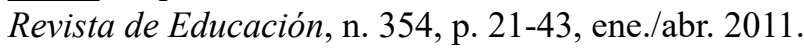

\section{Informações dos autores}

Sara Serrate González: Doctora en Educación. Profesora Ayudante Doctora. Facultad de Ciencias Sociales. Universidad de Salamanca. Contato: sarasg@usal.es.

iD http://orcid.org/0000-0002-9985-7623

Sonia Casillas Martín: Doctora en Pedagogía. Profesora Contratada Doctora. Facultad de Educación. Universidad de Salamanca. Contato: scasillasma@usal.es.

D http://orcid.org/0000-0001-5304-534X

Marcos Cabezas González: Doctor en Pedagogía. Profesor Contratado Doctor. Facultad de Educación. Universidad de Salamanca. Contato: mcabezasgo@usal.es.

iD http://orcid.org/0000-0002-3743-5839 